\title{
Free Fatty Acid is a Promising Biomarker in Triage Screening for Patients with Colorectal Cancer: A Case-Control Study
}

\author{
Bin Zhu (D) \\ Junrong Zhang $\mathbb{D}^{2}$ \\ Qingzhu Zheng (1) \\ Binhua Dong (D) 3 \\ Meihua Wang (D) \\ Jin Liu (D) \\ Yingping $\mathrm{Cao} \mathbb{D}^{\prime}$
}

'Department of Clinical Laboratory, Fujian Medical University Union Hospital, Fuzhou, 35000I, People's Republic of China; ${ }^{2}$ Department of Emergency Surgery, Fujian Medical University Union Hospital, Fuzhou, 35000I, People's Republic of China; ${ }^{3}$ Laboratory of Gynecologic Oncology, Fujian Provincial Maternity and Children's Hospital, Affiliated Hospital of Fujian Medical University, Fuzhou, 35000I, People's Republic of China
Correspondence: Yingping Cao Department of Clinical Laboratory, Fujian Medical University Union Hospital, No. 29, Xinquan Road, Fuzhou City, Fujian Province, People's Republic of China Email caoyingping9|8@sina.com
Purpose: The aim of our study was to identify the diagnostic ability of free fatty acids (FFAs) in younger colorectal cancer (CRC) patients by comparing carcinoembryonic antigen (CEA) and carbohydrate antigen 19-9 (CA19-9).

Methods: Patients screened for CRC at Fujian Medical University Union Hospital from January 2011 to December 2014 were recruited. Patients pathologically diagnosed with $\mathrm{CRC}$ or colorectal adenoma (CA) and healthy control participants were included. The enzyme endpoint method was applied to measure FFA levels. Receiver operating characteristic (ROC) curve analysis was performed to further evaluate the diagnostic ability of FFAs.

Results: FFA levels in late-stage patients (tumour-node-metastasis (TNM) stages IIIIV) were higher than those in early-stage patients (TNM stages I-II) ( $\mathrm{P}=0.02)$. The FFA levels in CRC patients were higher than those in controls of all ages, those younger than 50 years, males and females $(\mathrm{P}<0.001)$, and this difference was larger for patients younger than 50 years and females than for the all ages group. There was no significant difference in the FFA level between CA patients and healthy participants $(\mathrm{P}=0.53)$. The area under the curve (AUC) values of FFA, CEA, CA19-9, FFA+CEA, FFA+CA19-9 and FFA+CEA+CA19-9 distinguished CRC patients from controls at all ages, with values of $0.604,0.731,0.640,0.754,0.678$ and 0.758 , respectively; however, in the younger CRC patients (age $\leq 50$ ), the AUC values were $0.701,0.735,0.669,0.798$, 0.749 , and 0.801 . The AUC in female patients younger than 50 years was larger than that in males $(0.769$ vs 0.660$)$, and this value was greater than the value for CEA in males (0.739) and females (0.729).

Conclusion: The FFA level not only can complement the predictive ability of the CEA and CA19-9 levels but also has a superior predictive ability in female and younger patients with CRC. FFA levels may have a potential role in triage screening of early CRC.

Keywords: free fatty acid, colorectal cancer, colorectal adenoma, sex, younger patients

\section{Introduction}

Colorectal cancer (CRC) remains a common cancer worldwide and a significant public health burden. The estimated annual incidence is 1.2 million new cases per year. ${ }^{1}$ Unfortunately, while we have witnessed a declining incidence trend over the past few decades in the older population due to widespread colonoscopic screening, ${ }^{2}$ incidence and mortality rates for adults aged 20-49 have been increasing steadily. $^{3}$ On the one hand, younger patients with CRC suffer from 
clinicopathologic features and underlying tumour biology, such as methylation status, microsatellite instability, and somatic mutations, compared with those in their older counterparts. Colorectal tumours have more unfavourable histologic features, are usually diagnosed at a more advanced stage, and more frequently involve left-sided primary tumours. ${ }^{4,5}$ On the other hand, due to a lack of routine screening, emerging lifestyle issues such as obesity, lack of exercise, dietary factors and the widespread belief that cancer is associated with ageing, family doctors can too easily discount the possibility of colorectal cancer in young patients, even when they present with characteristic symptoms such as blood in the stool and abdominal pain. ${ }^{4,5}$ It was reported that the median time to treatment from symptom onset in CRC in patients younger than 50 years was more than 7 times that in older patients. ${ }^{6}$ It is critical to find a reliable biomarker to diagnose younger patients, who do not undergo colonoscopy screening, with CRC as early as possible.

Free fatty acids (FFAs) are triacylglycerol (TAG) precursors that are needed to replenish TAG stores in adipose, liver and muscle tissue through esterification; when hepatic glycogen is low and when muscles need energy, the TAGs in adipose tissue are broken down into FFAs for energy. ${ }^{7}$ FFAs are involved in insulin resistance and the metabolism of glucose and lipids and may play a role in the progression of diseases, such as diabetes, coagulation/ fibrinolysis disorders and hypertension. ${ }^{8-10}$ Recently, increasing evidence has demonstrated that cancer involves numerous metabolic pathways, such as glycolysis and the tricarboxylic acid cycle. ${ }^{11}$ In addition, cancer cells frequently exhibit alterations in fatty acid metabolism to sustain growth and proliferation, fulfil energy requirements and provide metabolites for anabolic processes, which can be represented by abnormal FFA levels. ${ }^{12}$

In our clinical work, we found that in some cancer patients, the FFA level was elevated even when TAG and total cholesterol (TCHO) levels were normal, indicating that a high FFA level may be the only abnormality in biochemical tests. To our knowledge, evidence about whether FFAs can be considered screening biomarkers is insufficient. In this study, we measured the FFA level in 1023 patients with $\mathrm{CRC}$ and 612 with colorectal adenoma (CA) to analyse the value of FFAs in the diagnosis of CRC according to patient age. To evaluate the diagnostic performance of FFA in younger CRC patients, patients under 50 years old were analysed. $^{13,14}$ Furthermore, we evaluated whether
FFAs have a potential role as prognostic biomarkers by evaluating the association between FFA levels and clinicopathologic characteristics in patients with CRC. Our study suggested that the FFA level has not only the ability to complement CEA and CA19-9 levels, but also has superior predictive ability in female and younger patients with CRC. Additionally, the FFA level has a potential role in prediction of tumour-nodemetastasis (TNM)stage III and IV.

\section{Materials and Methods Study Subjects}

This retrospective cohort consisted of healthy patients undergoing routine physical examination at the medical examination centre and inpatients who underwent colonoscopy biopsy and radical colorectal cancer resection at Fujian Medical University Union Hospital from January 2011 to December 2014. A total of 1023 patients pathologically diagnosed with CRC, 621 patients with $\mathrm{CA}$ and 330 healthy participants were recruited for our study. There were 210 men and 120 women among the healthy participants [median age (interquartile range, IQR): 59 (49-68) years], 399 men and 213 women among the patients with CA [median age (interquartile range, IQR): 60 (50-67) years], and 647 men and 376 women among the patients with CRC [median age (interquartile range, IQR): 59 (50-68) years] $(\mathrm{P}=0.214)$. Body mass index $(\mathrm{BMI})$ was similar in healthy participants [23.11 (19.92-25.91)] and in patients with CA [23.51 (19.79-25.87)] or CRC [23.91 (19.02-26.69)] $(\mathrm{P}=0.294)$. The CEA and CA19-9 levels in healthy individuals, CA patients and CRC patients were $1.95 \pm 1.03,3.21 \pm 6.64$, and $14.39 \pm 47.61 \mathrm{ng} / \mathrm{mL}$ and $10.50 \pm 6.69, \quad 15.58 \pm 42.60$, and $39.26 \pm 101.8 \mathrm{U} / \mathrm{mL}$, respectively. Patients with diabetes, hypertension, a family history of obesity, atherosclerosis, fatty liver, BMI $>27$ or other malignancies were excluded. The baseline characteristics of the study population are shown in Table 1. National and international professional societies from Europe and the United States have recommended starting colonoscopy screening from age 50 years, ${ }^{13,14}$ and CRC patients under age 50 were defined as young patients in our study. Patients with CRC were included in the case group, and patients with $\mathrm{CA}$ as well as healthy participants were included in the control group. 
Table I Baseline Characteristics of the Study Population Including Healthy Participants and Patients with Colorectal Adenomas and CRC in This Study

\begin{tabular}{|c|c|c|c|c|c|}
\hline Parameter & Normal $(n=330)$ & $C A(n=6 \mid 2)$ & \multicolumn{2}{|c|}{ CRC $(n=1023)$} & $P$ value \\
\hline Male & 210 & 399 & \multicolumn{2}{|c|}{647} & \multirow[t]{2}{*}{$0.724^{\mathrm{a}}$} \\
\hline Female & 120 & 213 & \multicolumn{2}{|c|}{376} & \\
\hline Age (Years), Median (Range) & $59(49-68)$ & $60(50-67)$ & \multicolumn{2}{|c|}{$59(50-68)$} & $0.24 I^{b}$ \\
\hline BMI (kg/m2), Median (Range) & 23.11 (19.92-25.91) & 23.5 I (19.79-25.87) & \multicolumn{2}{|c|}{23.91 (19.02-26.69) } & $0.294^{b}$ \\
\hline CEA (ng/mL), Mean \pm SD & $1.95 \pm 1.03$ & $3.21 \pm 6.64$ & \multicolumn{2}{|c|}{$|4.39 \pm 47.6|$} & $<0.001^{\mathrm{b}}$ \\
\hline CAI9-9 (U/mL), Mean \pm SD & $10.50 \pm 6.69$ & $15.58 \pm 42.60$ & \multicolumn{2}{|c|}{$39.26 \pm 101.8$} & $<0.00 \mathrm{I}^{\mathrm{b}}$ \\
\hline FFA (mmol/L) Mean \pm SD & $0.48 \pm 0.22$ & $0.49 \pm 0.22$ & \multicolumn{2}{|c|}{$0.58 \pm 0.27$} & $<0.001^{b}$ \\
\hline Tumour TNM stage (n, \%) & l & l & $\begin{array}{l}\text { I } \\
\text { II } \\
\text { III } \\
\text { IV }\end{array}$ & $\begin{array}{l}\mid 42 \text { (I3.9\%) } \\
236(23.1 \%) \\
47 \mid(46.0 \%) \\
\mid 74(17.0 \%)\end{array}$ & l \\
\hline Tumour location (n, \%) & l & 1 & $\begin{array}{l}\text { Colon } \\
\text { Rectum }\end{array}$ & $\begin{array}{l}388(37.9 \%) \\
635(62.1 \%)\end{array}$ & l \\
\hline Tumour sizes (n, \%) & 1 & 1 & $\begin{array}{l}<5 \mathrm{~cm} \\
\geq 5 \mathrm{~cm}\end{array}$ & $\begin{array}{l}370(36.2 \%) \\
653(63.8 \%)\end{array}$ & l \\
\hline Lymph node metastasis ( $\mathrm{n}, \%$ ) & l & l & $\begin{array}{l}\text { No } \\
\text { Yes }\end{array}$ & $\begin{array}{l}594(58.1 \%) \\
429 \text { (41.9\%) }\end{array}$ & I \\
\hline
\end{tabular}

Notes: ${ }^{a}$ The chi-square test was performed to analyse significant differences. ${ }^{\mathrm{b}}$ One-way ANOVA was performed to analyse significant differences.

Abbreviations: CA, colorectal adenoma; CRC, colorectal cancer; BMI, body mass index; CEA, carcinoembryonic antigen; CAI9-9, cancer antigen I9-9; TNM, tumournode-metastasis, SD, standard deviation.

Analysis of FFA, CEA, and CA 19-9 Levels

Peripheral blood samples were obtained from patients newly diagnosed with CRC. FFA levels were measured routinely with a Cobas 8000 automatic biochemical analyser (Roche, Diagnostics, Basel, Switzerland) according to the manufacturer's instructions. The enzyme endpoint method was applied to measure FFA levels. The FFAs that we detected were nonesterified fatty acids (Wako Pure Chemical Corporation, Japan). The serum levels of CEA and CA19-9 were measured using a Cobas 6000 analyser (Roche Diagnostics, Basel, Switzerland). According to the manufacturer's instructions, the cut-off value for normal CEA was $5 \mathrm{ng} / \mathrm{mL}$ and that for normal CA19-9 was 37 $\mathrm{U} / \mathrm{mL}$.

\section{Statistical Analysis}

Statistical analysis was performed using SPSS version 21.0 software or GraphPad Prism version 8.0. All measurement data were analysed to determine normality and the homogeneity of variance between the two groups. Data with a normal distribution and homogeneity of variance were analysed with Student's $t$ test to determine significant differences; otherwise, a nonparametric test was applied. One-way ANOVA was used to analyse significant differences among more than two groups, and the LSD- $t$ test was used for post hoc comparisons. The chi-square test was used to analyse the difference between males and females in normal, CA and CRC. The diagnostic value of FFA was estimated by receiver operating characteristic (ROC) curves. The Youden index was used to determine the optimal cut-off value for FFAs to differentiate between CRC patients and controls, and sensitivity and specificity were calculated according to the cut-off value. All tests were two-tailed, and a $\mathrm{P}$ value less than 0.05 was considered statistically significant. A logistic regression model was used to analyse the relationship between covariates and the probability of CRC in the case and control groups; the OR was adjusted for covariates. 


\section{Results}

\section{FFA Levels in CRC Patients and the Age Range of Patients with CA and CRC}

The concentrations of FFAs in the peripheral blood of healthy participants, patients with $\mathrm{CA}$, and patients with CRC were $0.483 \pm 0.217 \mathrm{mmol} / \mathrm{L}, \quad 0.492 \pm 0.217$ $\mathrm{mmol} / \mathrm{L}$, and $0.579 \pm 0.268 \mathrm{mmol} / \mathrm{L}$, respectively. The FFA level in patients with CRC was higher than that in CA patients $(\mathrm{P}<0.001)$ and healthy participants $(\mathrm{P}<0.001)$. There was no significant difference in FFA levels between CA patients and healthy participants $(\mathrm{P}=0.53)$. The FFA levels in healthy, $\mathrm{CA}$ and $\mathrm{CRC}$ patients are shown in Table 1.

By recruiting CRC patients treated in our hospital for four years, $26.3 \%(269 / 1023)$ of patients with CRC younger than 50 years old, $9.7 \%(100 / 1023)$ younger than 45 years old, and $5.5 \%(56 / 1023)$ younger than 40 years old were included in our study. In addition, $25.3 \%(155 / 612)$ of patients with CA younger than 50 years old, $14.5 \%(89 / 612)$ younger than 45 years old, and $8.5 \%(52 / 612)$ younger than 40 years old were finally included.

\section{Association Between FFA Levels and Clinicopathological Characteristics in CRC Patients}

In the analysis of the clinicopathological data of CRC patients, we found that the FFA level in TNM stage III + IV patients was higher than that in TNM stage I+II patients $(0.60 \pm 0.38$ vs $0.56 \pm 0.24, \mathrm{mmol} / \mathrm{L}, \mathrm{P}=0.02)$, but the FFA level was not significantly correlated with tumour location, tumour size, lymph node metastasis, CEA level or CA19-9 level in the peripheral blood.
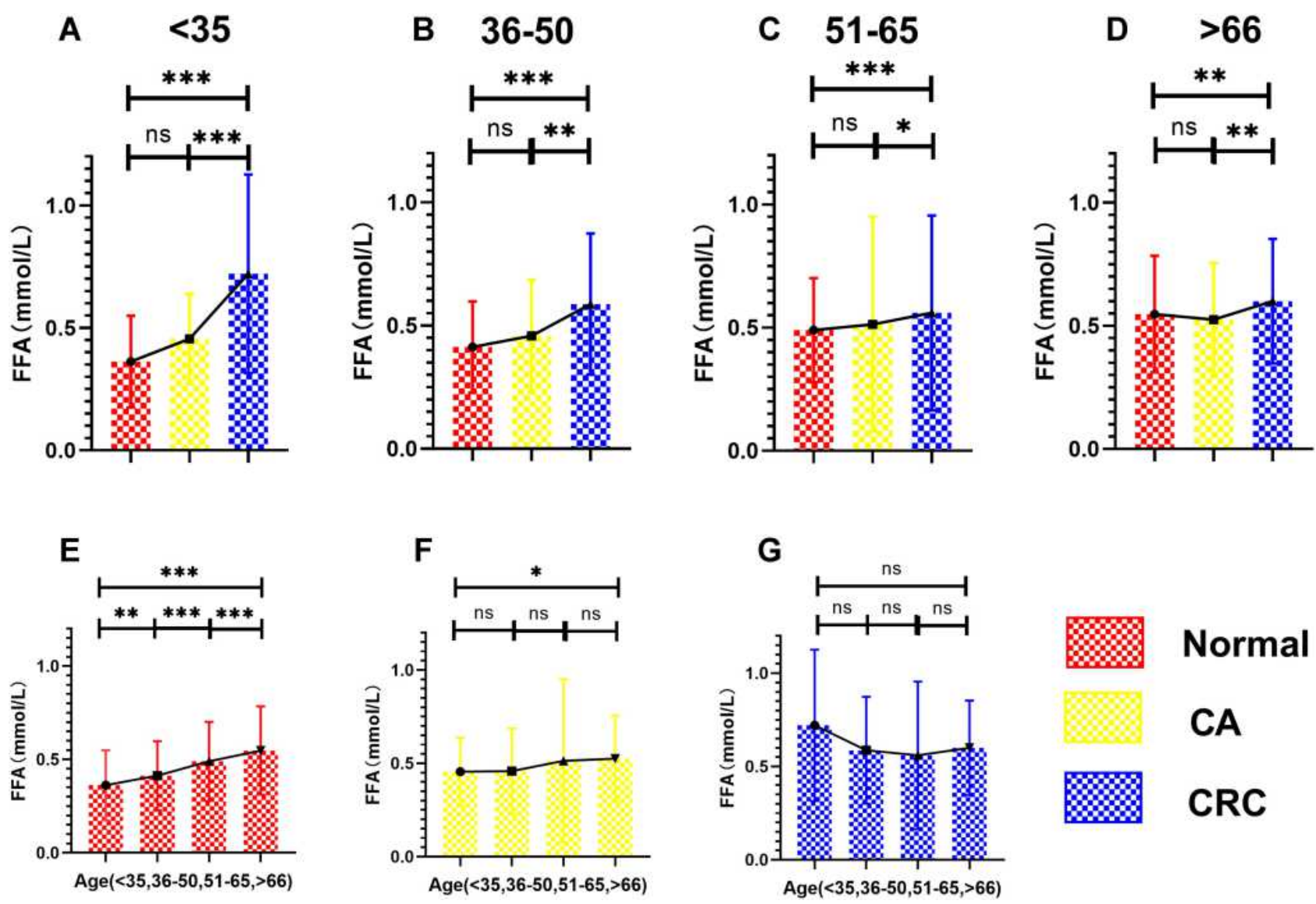

Normal

Figure I FFA levels in controls and CRC patients according to age ( $<35,36-50,51-65$, and $>66$ years). The differences between groups (normal vs CA, normal vs CRC and CA vs CRC) were analysed by Student's $t$ test. One-way ANOVA was used to analyse significant differences among the normal, CA and CRC groups. The LSD- $t$ test was used for post hoc comparisons of different age groups. *, <0.05; **, <0.0I; ***, <0.00I. A-D: FFA level differences between Normal, CA and CRC in groups of Age $<35$, Age $_{36-50}$, Age ${ }_{51-65}$, Age $>66$. E-G: FFA level differences from patients younger than age 35 to older than 66 in groups of Normal, CA and CRC.

Abbreviations: CA, colorectal adenoma; CRC, colorectal cancer; Ns, no significant difference. 


\section{FFA Levels in Healthy Participants and Patients with CA and CRC of Different Ages}

To investigate the relationship between FFA levels and age in detail, we divided the healthy participants and patients with $\mathrm{CA}$ and $\mathrm{CRC}$ into four groups according to the average age distribution. As shown in Figure 1, the FFA levels increased with age in normal participants $(\mathrm{P}=0.001)$ and patients with $\mathrm{CA}(\mathrm{P}=0.032)$ but did not increase with age in CRC $(\mathrm{P}=0.075)$. The FFA level increased with age in the controls $(\mathrm{P}=0.001)$ and with colorectal disease severity in the age $\leq 35(\mathrm{P}<0.001)$, age $36-50(\mathrm{P}<0.001)$, age $51-65(\mathrm{P}=0.008)$, and age $>66(\mathrm{P}=0.002)$ groups, and as age increased, the curve flattened in CRC patients compared with controls.

\section{Evaluation of FFA as a Potential Diagnostic Biomarker for CRC from 2I to 88 Years Old}

The area under the curve (AUC) values for FFA, CEA and CA19-9 were 0.604, 0.731 and 0.640, respectively. For combined detection, the AUCs of FFA+CEA, FFA +CA19-9, and FFA+CEA+CA19-9 were 0.754, 0.678 and 0.758 , respectively (Figure 2). As shown in Table 2, the sensitivities of FFA, CEA and CA19-9 were 41.6, 55.8 and 45.7, and the sensitivities of the combinations were 49.5, 50.3 and 53.9. The specificities of FFA, CEA and CA19-9 were 73.1, 81.1 and 77.3, respectively. For combined detection, the specificities were 90.4, 75.8 and 87.3.

\section{Evaluation of FFA as a Potential Diagnostic Biomarker for CRC Younger Than or Equal to 50 Years Old}

The AUC values for FFA, CEA and CA19-9 were $0.701,0.735$ and 0.669 , respectively. For combined detection, the AUCs of FFA+CEA, FFA+CA19-9, and FFA+CEA+CA19-9 were 0.798, 0.749 and 0.801, respectively (Figure 3). As shown in Table 3, the sensitivities of FFA, CEA and CA19-9 were 48.7, 50.4 and 48.3 , and the sensitivities of the combinations were 59.2, 67.4 and 68.2. The specificities of FFA, CEA and CA19-9 were 84.5, 87.5 and 81.6, respectively. For combined detection, the specificities were 81.8, 79.1 and 79.3.
Performance and Cutoff Values of Three

Biomarkers for Determining the Incidence of CRC in the Case and Control Groups in Patients Younger or Older Than 50 Years

According to the multivariate logistic regression model analysis (Table 4), the prognostic values for CRC of FFA, CEA and CA19-9 in patients younger than 50 years old were 5.210, 7.068 and 4.298, and those for patients older than 50 years were $1.421,5.073$ and 2.533 .

\section{Diagnostic Performance of FFA in Distinguishing CRC Patients from Controls by Sex}

As shown in Figure 4, significant differences were observed in both males and females to distinguish patients with CRC from controls $(\mathrm{P}<0.001)$, and little diagnostic value was found between the $\mathrm{CA}$ and normal groups. When we detected FFA levels in normal adults, we found that FFA levels were elevated in females compared with males $(\mathrm{P}<0.05)$. Interestingly, when colorectal severity worsened, the growth of FFAs in females was larger than that in males with CRC. The AUCs to diagnose CRC from controls in females and males were 0.642 and 0.587 , and the AUCs in females and males younger than 50 were 0.769 and 0.660 , respectively.

\section{Discussion}

In the present study, we found that the ability of the AUC of FFA levels in combination with CEA and CA19-9 to distinguish CRC patients was 0.801 , and the ability of the AUC to distinguish female patients with CRC younger than 50 years was 0.769 , indicating that FFA levels not only can complement the predictive ability of CEA and CA19-9, but also have a superior predictive ability in female and younger patients with CRC. National and international professional societies from Europe and the United States have recommended starting colonoscopy screening from age 50 years, ${ }^{13,14}$ so CRC patients under age 50 were defined as young patients in our study. On the one hand, early-onset $\mathrm{CRC}$ is characterized by a more advanced stage, poorly differentiated tumours, mucinous neoplasms, a more distal location, and a unique biomarker profile, while survival outcomes for young patients even exceed those of older patients. ${ }^{4,5}$ On the other hand, younger CRC patients are a patient population where the 

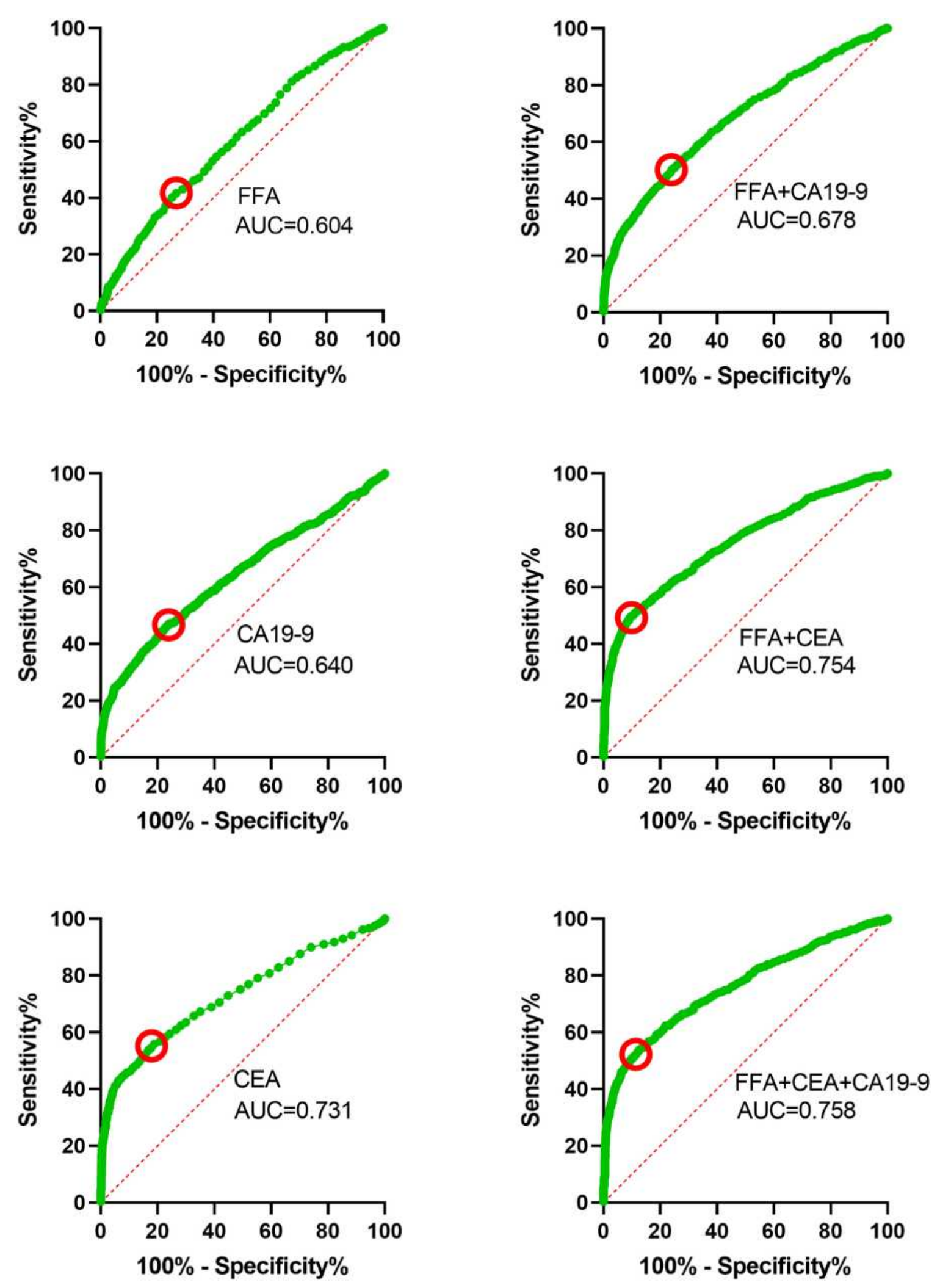

Figure 2 ROC curves of single and combined detection with FFA, CEA and CAI9-9 in patients 21 to 88 years old. ROC curve analysis was used to evaluate the ability to distinguish patients with CRC from controls. Patients with CA and healthy participants were considered as controls.

Abbreviations: ROC, receiver operating characteristic; AUC, area under the curve; CEA, carcinoembryonic antigen; CAI9-9, carbohydrate antigen I9-9. 
Table 2 The Values of FFA, CEA, and CAI9-9 Alone and Combined Biomarkers for Distinguishing CRC Patients from Healthy Participants and Patients with Colorectal Adenomas from 21 to 88 Years Old

\begin{tabular}{|l|c|c|c|c|c|c|}
\hline Variables & AUC & Cut-Off & Sensitivity\% & Specificity\% & \multicolumn{2}{|c|}{$95 \%$ Confidence Interval } \\
\cline { 3 - 7 } & & & & & Lower Limit & Upper Limit \\
\hline FFA & 0.604 & 0.585 & 41.6 & 73.1 & 58.2 & 62.6 \\
CAI9-9 & 0.640 & 37 & 45.7 & 77.3 & 61.8 & 71.0 \\
CEA & 0.731 & 5 & 55.8 & 81.1 & 65.7 & 75.1 \\
FFA+CAI9-9 & 0.678 & 1 & 50.3 & 75.8 & 69.9 \\
FFA+CEA & 0.754 & 1 & 49.5 & 90.4 & 73.5 & 77.4 \\
FFA+CEA+CA19-9 & 0.758 & $/$ & 53.9 & 87.3 & 73.9 & 77.8 \\
\hline
\end{tabular}

Abbreviation: AUC, area under the curve.

use of screening is limited; because physicians often believe CRC is an age-related disease, critical symptoms often go unrecognized. ${ }^{5,6}$ It is critical to identify a reliable biomarker to diagnose younger patients, who do not undergo colonoscopy screening, with CRC as early as possible. CEA and CA19-9 are well-known serological tumour markers associated with $\mathrm{CRC}$, and they are both overexpressed in CRC tissues. ${ }^{15,16}$ Both CEA and CA19-9 levels in serum are affected by the progression of cancer tissues and whether they are released into the blood, whose detection ability and influencing factors are relatively consistent.

FFAs are not only an important energy substrate for a number of organs but are also TAG precursors that are needed to replenish TAG stores in adipose, liver and muscle tissue through esterification. ${ }^{7}$ When hepatic glycogen is low and when muscles need energy, the TAGs in adipose tissue are broken down into FFAs for energy. Some studies have focused on the potential role of FFAs in CRC. Al Mahrietal ${ }^{17}$ and Bartoszeketal ${ }^{18}$ demonstrated that the free fatty acid receptor 2 and 3 (FFAR2/FFAR3) genes, which are closely associated with FFA metabolism, promote the proliferation of colon cancer cells; in addition, treatment through the target of FFARs may be helpful to CRC. Moreover, Kawaguchi et $\mathrm{al}^{19}$ and Hsiao et $\mathrm{al}^{20}$ demonstrated that high expression of fatty acid-binding protein 5 (FABP5) promotes cell growth and metastasis in CRC and that pterostilbene inhibits CRC cell migration induced by adipocyte-conditioned medium by targeting the FABP5-related signalling pathway. These findings indicated that FFAs are involved in the progression of CRC and that FFAs may play an important role in energy metabolism stimulated by CRC tissues, consistent with our study. Moreover, Zhang ${ }^{21}$ et al demonstrated that the serum levels of FCH (free cholesterol) were significantly higher in patients with colorectal cancer than in patients with benign colorectal disease and healthy participants, which indicated that $\mathrm{FCH}$ has a potential role in the early diagnosis of CRC, consistent with our results; however, the FFA level was not detected in Xin Zhang's study, ROCs of FCH and other lipids were not drawn, and the diagnostic performance of FCH was unclear. In interest, Zhang $^{22}$ et al detected FFA levels to investigate whether FFAs are meaningful in cancer patients and found that AUCs in rectal cancer and colon cancer distinguishing noncancer patients (without patients with CA) were 0.614 and 0.599, respectively, which indicated a comparatively general diagnostic value; however, it was found that FFAs not only have the value of early diagnosis of CRC but also have good complementary efficacy with CEA and CA19-9 (expressed in CRC tissues), and the combined detection can improve the early diagnostic ability in our study, the AUC of which is 0.801 and larger than Lili Zhang's result.

CRC screening guidelines do not distinguish females from males, but there are technical limitations of endoscopic examinations in females, who have longer average total and transverse colon lengths, more frequent occurrence of flat-type right-sided colon cancer and a narrower colon diameter. In addition, socio-cultural barriers can also delay screening and diagnosis in female patients. ${ }^{23}$ In our study, we found that the FFA levels of women were better than those of men in distinguishing CRC patients, but the efficacy was relatively weaker. However, when the performance of early diagnosis in young patients under the age of 50 was studied, we found that the diagnostic value of FFAs in women was significantly 

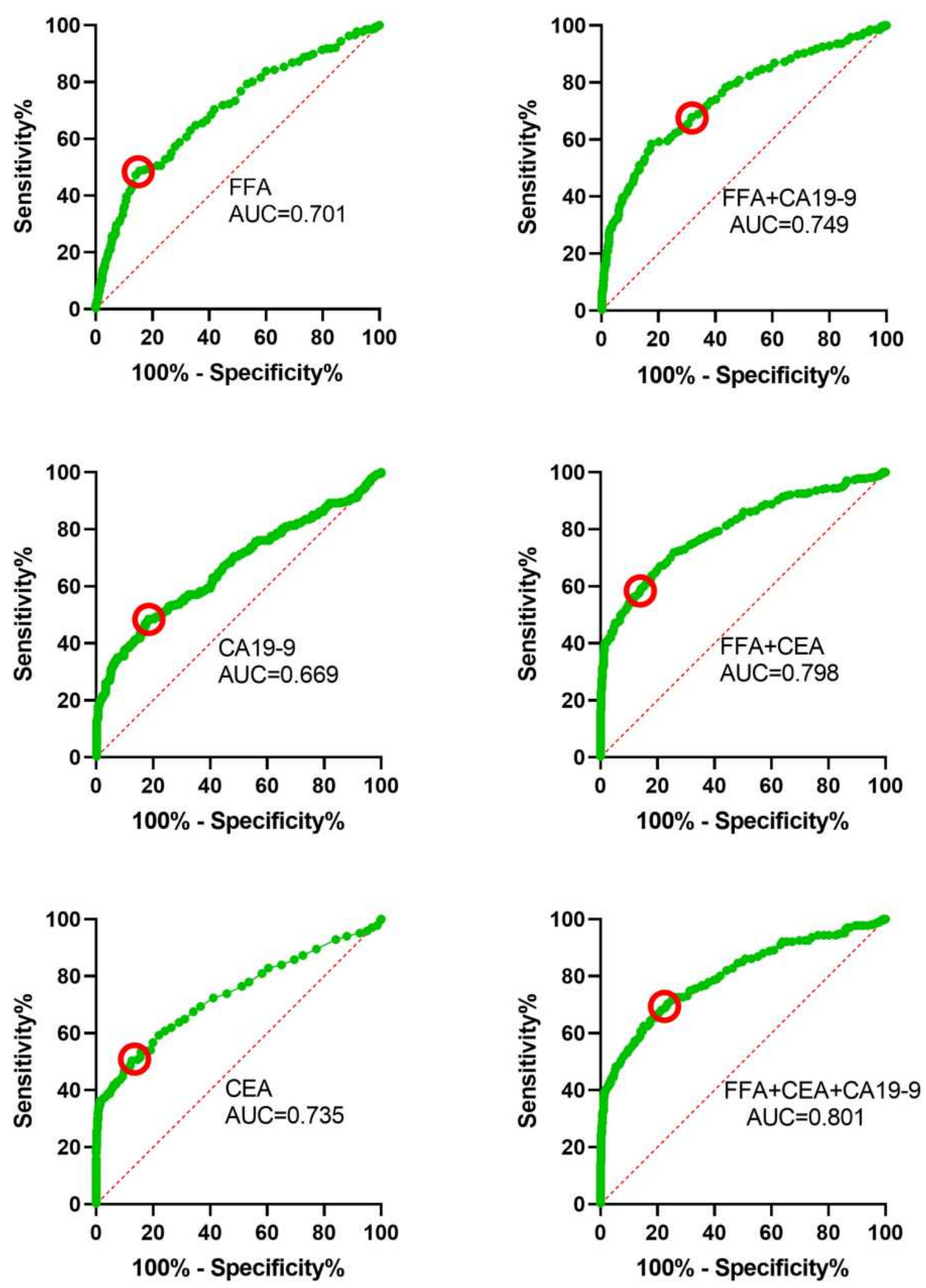

Figure 3 ROC curves for single and combined detection with FFA, CEA and CAI9-9 in patients younger than 50 years old. ROC curve analysis was used to evaluate the ability to distinguish patients with CRC from controls among those younger than 50 years. Patients with CA and healthy participants were considered as controls. 
Table 3 The Values of FFA, CEA, and CAI9-9 Alone and Combined Biomarkers for Distinguishing CRC Patients from Healthy Participants and Patients with Colorectal Adenomas Younger Than or Equal to 50 Years Old

\begin{tabular}{|l|c|c|c|c|c|c|}
\hline Variables & AUC & Cut-Off & Sensitivity\% & Specificity\% & \multicolumn{2}{|c|}{ 95\% Confidence Interval } \\
\cline { 3 - 7 } & & & & & Lower Limit & Upper Limit \\
\hline FFA & 0.701 & 0.585 & 48.7 & 84.5 & 66.1 & 74.1 \\
CAI9-9 & 0.667 & 37 & 48.3 & 81.6 & 62.4 & 71.1 \\
CEA & 0.734 & 5 & 50.4 & 87.5 & 69.3 & 77.4 \\
FFA+CA19-9 & 0.749 & 1 & 67.4 & 79.1 & 7.1 & 78.7 \\
FFA+CEA & 0.798 & 1 & 59.2 & 79.3 & 83.4 & 76.5 \\
FFA+CEA+CA19-9 & 0.801 & 1 & 68.2 & 79.3 & 83.6 \\
\hline
\end{tabular}

Abbreviation: AUC, area under the curve.

Table 4 Ability of Serological Biomarkers to Predict CRC in Patients Younger or Older Than 50 Years

\begin{tabular}{|c|c|c|c|c|c|}
\hline \multirow[t]{2}{*}{ Variables } & \multirow[t]{2}{*}{ No. of Patients } & \multirow[t]{2}{*}{ Cutoff Value } & \multicolumn{3}{|c|}{ Multivariate Analysis } \\
\hline & & & $\mathrm{OR}_{\text {ajusted }}{ }^{\mathrm{a}}$ & $95 \% \mathrm{Cl}$ & $P$ value \\
\hline \multicolumn{6}{|c|}{ Younger than 50 years old } \\
\hline FFA (mmol/L) & $\begin{array}{l}367 \\
144\end{array}$ & $\begin{array}{l}<0.585 \\
>0.585\end{array}$ & $\begin{array}{l}\mathrm{I}(\mathrm{R}) \\
5.210\end{array}$ & $\begin{array}{l}- \\
3.661-7.413\end{array}$ & 0.001 \\
\hline CEA $(\mathrm{ng} / \mathrm{mL})$ & $\begin{array}{l}375 \\
136\end{array}$ & $\begin{array}{l}<5 \\
>5\end{array}$ & $\begin{array}{l}\mathrm{I}(\mathrm{R}) \\
7.068\end{array}$ & $\begin{array}{l}- \\
4.879-10.239\end{array}$ & 0.001 \\
\hline CAI9-9 (U/mL) & $\begin{array}{l}364 \\
147\end{array}$ & $\begin{array}{l}<37 \\
>37\end{array}$ & $\begin{array}{l}I(R) \\
4.298\end{array}$ & $\begin{array}{l}- \\
3.039-6.080\end{array}$ & 0.001 \\
\hline \multicolumn{6}{|c|}{ Older than 50 years old } \\
\hline FFA (mmol/L) & $\begin{array}{l}944 \\
510\end{array}$ & $\begin{array}{l}<0.585 \\
>0.585\end{array}$ & $\begin{array}{l}\mathrm{I}(\mathrm{R}) \\
\mathrm{I} .42 \mathrm{I}\end{array}$ & $\begin{array}{l}- \\
1.174-1.720\end{array}$ & 0.001 \\
\hline CEA $(\mathrm{ng} / \mathrm{mL})$ & $\begin{array}{l}896 \\
558\end{array}$ & $\begin{array}{l}<5 \\
>5\end{array}$ & $\begin{array}{l}\mathrm{I}(\mathrm{R}) \\
5.073\end{array}$ & $\begin{array}{l}- \\
4.151-6.199\end{array}$ & 0.001 \\
\hline CAI9-9 (U/mL) & $\begin{array}{l}977 \\
477\end{array}$ & $\begin{array}{l}<37 \\
>37\end{array}$ & $\begin{array}{l}\text { I (R) } \\
2.533\end{array}$ & $\begin{array}{l}- \\
2.085-3.076\end{array}$ & 0.001 \\
\hline
\end{tabular}

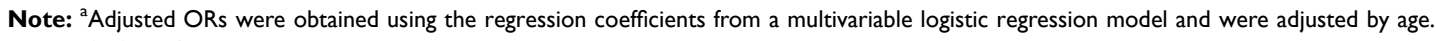
Abbreviation: OR, odds ratio.

better than that in men, the AUC of which was 0.769, which is larger than Lili Zhang's result and larger than the AUC value of CEA in female patients (0.729).

The following limitations of our study must be considered. First, FFA experiments should be performed in vitro and in vivo to investigate the role of FFAs in $\mathrm{CRC}$, and the survival status of patients with CRC should be investigated to assess the prognostic value of FFA assessment. Second, some groups had relatively small sample sizes such as patients younger than Age 35 , and it might raisebias. Third, as references of lipids and lipoproteins vary by age and sex, ${ }^{24}$ the short followup duration of the study with a single-centred retrospective design might raise bias towards sample selection and analysis. Hence, further prospective multicentre studies are needed to validate the clinical significance of FFA in patients with CRC.

\section{Conclusion}

In summary, the FFA level can not only complement the predictive ability of CEA and CA19-9, but also has a superior predictive ability in female and younger patients with CRC. In addition, the FFA level can distinguish CRC patients with TNM stage III and IV from those with stage I and II. The FFA level may have a potential role in triage screening of early CRC and in secondary prevention. 


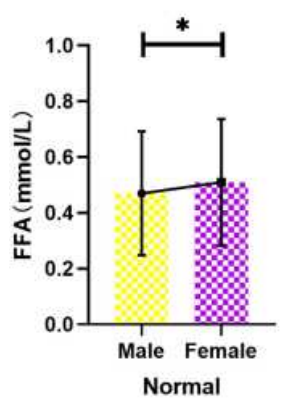

CEA (Male and <50)

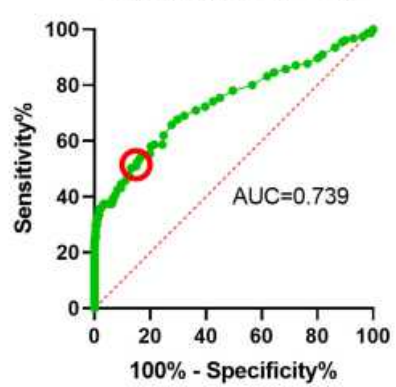

FFA (Male and <50)

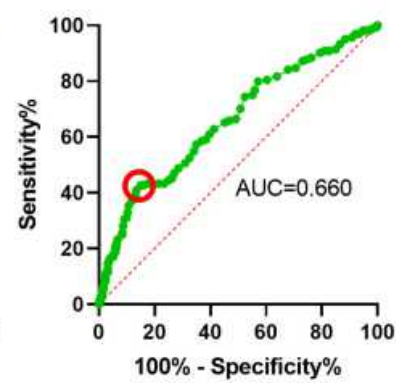

CEA (Female and <50)

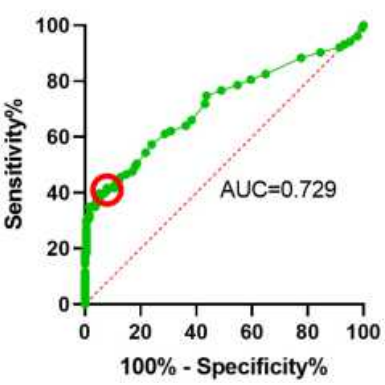

FFA (Female and $<50$ )

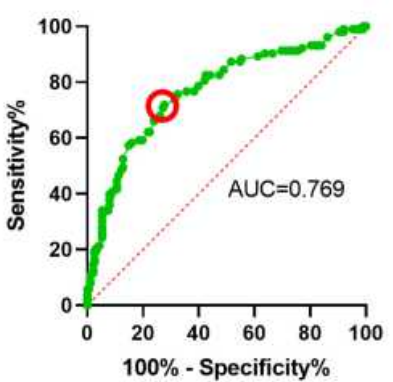

Figure 4 FFA levels in controls and CRC patients according to sex; ROC curves of FFA and CEA in patients younger than 50 years according to sex. The differences between groups (normal vs CA, normal vs CRC and CA vs CRC) were analysed by Student's $t$ test. The ROC curve of CEA and FFA was used to evaluate the ability to distinguish patients with CRC from controls among patients younger than 50 years. Patients with CA and healthy participants were considered as controls. *, $<0.05$; $* * *,<0.00 \mathrm{I}$.

Abbreviation: Ns, no significant difference.

\section{Abbreviations}

FFA, free fatty acid; CEA, carcinoembryonic antigen; CA19-9, carbohydrate antigen 19-9; FFAR, free fatty acid receptor; FABP, fatty acid-binding protein; CRC, colorectal cancer; TAG, triacylglycerol; AUC, area under the curve; $\mathrm{CA}$, colorectal adenoma.

\section{Ethics Statement}

This study was carried out in accordance with the World Medical Association Declaration of Helsinki. This study received a documented review and approval from a formally constituted review board (the ethics committee of Fujian Medical University Union Hospital, 2020WSJTK063). All patients and healthy volunteers provided written informed consent prior to their inclusion in the study.

\section{Acknowledgments}

The authors would like to thank Fujian Medical University Union Hospital for providing the clinical data. Above all, we are grateful to the patients who made the study possible.

\section{Author Contributions}

All authors contributed to data analysis, drafting or revising the article, have agreed on the journal to which the article will be submitted, gave final approval of the version to be published, and agree to be accountable for all aspects of the work.

\section{Funding}

The Young and Middle-aged Teachers Fund, Education Department of Fujian Province, No. JT180176; the Startup Fund for Scientific Research, Fujian Medical University, No. 2019QH1038; the Joint Funds for the Innovation of Science and Technology, Fujian Province, No. 2017Y9051, 2018Y9006; the Fujian Medicine Innovation Program, 2018-CX-18; the Fujian Undergraduate Education and Teaching Reform Project, No. FBJG20180225 and the Key Project of Educational Reform in Fujian Medical University, No. J18002.

\section{Disclosure}

The authors report no conflicts of interest regarding this work. 


\section{References}

1. Brenner H, Kloor M, Pox CP. Colorectal cancer. Lancet. 2014;383 (9927):1490-1502. doi:10.1016/s0140-6736(13)61649-9

2. Doubeni CA, Corley DA, Quinn VP, et al. Effectiveness of screening colonoscopy in reducing the risk of death from right and left colon cancer: a large community-based study. Gut. 2018;67(2):291-298. PMID: 27733426 PMCID: PMC5868294. doi:10.1136/gutjnl-2016312712

3. Siegel RL, Miller KD, Fedewa SA, et al. Colorectal cancer statistics, 2017. CA Cancer J Clin. 2017;67(3):177-193. PMID: 28248415. doi:10.3322/caac. 21395

4. Connell LC, Mota JM, Braghiroli MI, Hoff PM. The rising incidence of younger patients with colorectal cancer: questions about screening, biology, and treatment. Curr Treat Options Oncol. 2017;18(4):23. PMID: 28391421. doi:10.1007/s11864-017-0463-3

5. Mauri G, Sartore-Bianchi A, Russo AG, Marsoni S, Bardelli A, Siena S. Early-onset colorectal cancer in young individuals. Mol Oncol. 2019;13(2):109-131. PMID: 30520562 PMCID: PMC6360363. doi:10.1002/1878-0261.12417

6. Scott RB, Rangel LE, Osler TM, Hyman NH. Rectal cancer in patients under the age of 50 years: the delayed diagnosis. $\mathrm{Am}$ J Surg. 2016;211(6):1014-1018. PMID: 26651969. doi:10.1016/j. amjsurg.2015.08.031

7. Stich V, Berlan M. Physiological regulation of NEFA availability: lipolysis pathway. Proc Nutr Soc. 2004;63(2):369-374. PMID: 15294057. doi:10.1079/PNS2004350

8. Arner P. Insulin resistance in type 2 diabetes: role of fatty acids. Diabetes Metab Res Rev. 2002;18(Suppl 2):S5-9. PMID: 11921432. doi:10.1002/dmrr.254

9. Rui L. Energy metabolism in the liver. Compr Physiol. 2014;4 (1):177-197. PMID: $24692138 \quad$ PMCID: PMC4050641. doi:10.1002/cphy.c130024

10. Xiong $\mathrm{Z}, \mathrm{Xu} \mathrm{H}$, Huang $\mathrm{X}$, et al. Nonesterified fatty acids and cardiovascular mortality in elderly men with CKD. Clin J Am Soc Nephrol. 2015;10(4):584-591. PMID: 25637632 PMCID: PMC4386258. doi: $10.2215 /$ CJN.08830914

11. Anderson NM, Mucka P, Kern JG, Feng H. The emerging role and targetability of the TCA cycle in cancer metabolism. Protein Cell. 2018;9(2):216-237. PMID: 28748451 PMCID: PMC5818369. doi:10.1007/s13238-017-0451-1

12. Li J, Condello $\mathrm{S}$, Thomes-Pepin $\mathrm{J}$, et al. Lipid desaturation is a metabolic marker and therapeutic target of ovarian cancer stem cells. Cell Stem Cell. 2017;20(3):303-314 e305. PMID: 28041894 PMCID: PMC5337165. doi:10.1016/j.stem.2016.11.004

13. Qaseem A, Crandall CJ, Mustafa RA, Hicks LA, Wilt TJ; Clinical Guidelines Committee of the American College of P. Screening for colorectal cancer in asymptomatic average-risk adults: a guidance statement from the American College of Physicians. Ann Intern Med. 2019;171(9):643-654. PMID: 31683290. doi:10.7326/M190642
14. Pox C, Aretz S, Bischoff SC, et al. [S3-guideline colorectal cancer version 1.0]. Z Gastroenterol. 2013;51(8):753-854. German. PMID: 23955142. doi: $10.1055 / \mathrm{s}-0033-1350264$

15. Hammarstrom S. The carcinoembryonic antigen CEA family: structures, suggested functions and expression in normal and malignant tissues. Semin Cancer Biol. 1999;9:67•81. PMID: 10202129. doi:10.1006/scbi.1998.0119

16. Morales-Gutiérrez C, Vegh I, Colina F, et al. Survival of patients with colorectal carcinoma: possible prognostic value of tissular carbohydrate antigen 19.9 determination. Cancer. 1999;86(9):1675-1681. PMID: $\quad 10547539$ doi:10.1002/(SICI)1097-0142(19991101) 86:9<1675::AID-CNCR8 $>3.0$. CO $; 2-9$

17. AlMahri S, Al Ghamdi A, Akiel M, Al Aujan M, Mohammad S, Aziz MA. Free fatty acids receptors 2 and 3 control cell proliferation by regulating cellular glucose uptake. World J Gastrointest Oncol. 2020;12(5):514-525. PMID: 32461783 PMCID: PMC7235185. doi:10.4251/wjgo.v12.i5.514

18. Bartoszek A, Fichna J, Tarasiuk A, et al. Free fatty acid receptors as new potential targets in colorectal cancer. Curr Drug Targets. 2020;21(14):1397-1404. PMID: 31721710. doi:10.2174/ 1389450120666191112141901

19. Kawaguchi K, Senga S, Kubota C, Kawamura Y, Ke Y, Fujii H. High expression of fatty acid-binding protein 5 promotes cell growth and metastatic potential of colorectal cancer cells. FEBS Open Bio. 2016;6(3):190-199. PMID: 27047747 PMCID: PMC4794781. doi:10.1002/2211-5463.12031

20. Hsiao YH, Chen NC, Koh YC, Nagabhushanam K, Ho CT, Pan MH. Pterostilbene inhibits adipocyte conditioned-medium-induced colorectal cancer cell migration through targeting FABP5-related signaling pathway. J Agric Food Chem. 2019;67(37):10321-10329. PMID 31419115. doi:10.1021/acs.jafc.9b03997

21. Zhang X, Zhao XW, Liu DB, et al. Lipid levels in serum and cancerous tissues of colorectal cancer patients. World $J$ Gastroenterol. 2014;20(26):8646-8652. PMID: 25024621 PMCID: PMC4093716. doi:10.3748/wjg.v20.i26.8646

22. Zhang L, Han L, He J, Lv J, Pan R, Lv T. A high serum-free fatty acid level is associated with cancer. $J$ Cancer Res Clin Oncol. 2020;146(3):705-710. PMID: 31773260 PMCID: PMC7039835. doi:10.1007/s00432-019-03095-8

23. Kim SE, Paik HY, Yoon H, Lee JE, Kim N, Sung MK. Sex- and gender-specific disparities in colorectal cancer risk. World $J$ Gastroenterol. 2015;21(17):5167-5175. PMID: 25954090 PMCID: PMC4419057. doi:10.3748/wjg.v21.i17.5167

24. Balder JW, de Vries JK, Nolte IM, Lansberg PJ, Kuivenhoven JA, Kamphuisen PW. Lipid and lipoprotein reference values from 133,450 Dutch Lifelines participants: age- and gender-specific baseline lipid values and percentiles. J Clin Lipidol. 2017;11(4):10551064 e1056. PMID: 28697983. doi:10.1016/j.jacl.2017.05.007
Cancer Management and Research is an international, peer-reviewed open access journal focusing on cancer research and the optimal use of preventative and integrated treatment interventions to achieve improved outcomes, enhanced survival and quality of life for the cancer patient.
The manuscript management system is completely online and includes a very quick and fair peer-review system, which is all easy to use. Visit http://www.dovepress.com/testimonials.php to read real quotes from published authors. 